\title{
Autoridad y autoritarismo, una dicotomía en el salón de clases
}

\author{
Authority and authoritarianism, a dichotomy in the classroom
}

\section{Guadalupe Zuñe Flores}

Universidad Nacional Pedro Ruiz Gallo - Perú

Lambayeque, Perú

gzunef@unrg.edu.pe

Josué Mejía Guevara

Universidad Nacional Pedro Ruiz Gallo - Perú

Lambayeque, Perú

jmejiagu@unprg.edu.pe

\section{Lizeth Caramantin Castillo}

Universidad Nacional Pedro Ruiz Gallo - Perú Lambayeque, Perú

Icaramantin@unprg.edu.pe

Beder Bocanegra Vilcamango

Universidad Nacional Pedro Ruiz Gallo - Perú

Lambayeque, Perú

bbocanegra@unprg.edu.pe

\section{RESUMEN}

En el proceso formativo de la educación peruana, los estudiantes de Educación Básica Regular (EBR) han sentido que el salón de clases es el espacio donde se debe inclinar la cabeza y acatar órdenes del docente, siendo esta una evidente manifestación de autoritarismo, hecho que ha determinado el siguiente cuestionamiento: ¿Cuál es el constructo teórico que difiere conceptualmente el autoritarismo y la autoridad en el contexto de una relación dicotómica, entre maestro y estudiante? El estudio busca analizar la relación dicotómica entre el adecuado ejercicio de autoridad y el autoritarismo en el salón de clases. La metodología empleada es la Chakana Pregunta como estrategia didáctica para desarrollar el pensamiento crítico, la misma que consiste en elaborar nueve pregunta como producto de la lectura de un texto, con las nueve preguntas se elabora un texto argumentativo. Se trata de un estudio experimental que se basa en el análisis de un caso de maltrato escolar; para ello, se plantearon nueve preguntas y se ubicaron estratégicamente en la Chakana Pregunta, considerando el análisis vertical central, con la finalidad de desarrollar el artículo en función de las tres preguntas seleccionadas. El estudio ha permitido analizar y cuestionar el comportamiento del docente desde la práctica del autoritarismo y la autoridad según el contexto. El hallazgo es significativo, ya que ambas categorías asociadas al poder y castigo configuran el rol del docente cuando encara el proceso formativo. La aplicación de la Chakana Pregunta, como estrategia didáctica para redactar textos académicos ha sido muy útil porque ha permitido analizar el caso mediante tres preguntas dispuestas.

Palabras claves: Autoridad-autoritarismo; maestro-estudiante; escuela; chakana-pregunta; estrategia 


\section{ABSTRACT}

The training process within the Peruvian education system has many limitations centered on teacher performance, so the problems are varied and multicausal. Students in Regular Basic Education (EBR) have felt that a classroom is a place where they must bow their heads and obey orders from the person in charge of their education, which is an evident manifestation of authoritarianism. This fact has determined the following question: What is the theoretical construct that conceptually discriminates authoritarianism and authority in the context of a dichotomous relationship? The study seeks to analyze the dichotomous relationship between the proper exercise of authority and authoritarianism in the classroom. The methodology used is the Chakana pregunta as a didactic strategy to develop critical thinking. It is an experimental study based on the analysis of a case of school mistreatment; to this end, nine questions were posed and strategically placed in the Chakana pregunta, considering the central vertical analysis, with the aim of developing the article according to the three selected questions. The study has made it possible to analyze and question the teacher's behavior from the perspective of the practice of authoritarianism and authority according to the context. The finding is significant, as both categories associated with power and punishment shape the role of the teacher when facing the educational process. The application of the Chakana pregunta, as a didactic strategy for writing academic texts, has been very useful because it has made it possible to analyze the case by means of three questions.

Keywords: Authority-authoritarianism; teacher-student; Chakana pregunta; strategy

\section{INTRODUCCIÓN}

La escuela, dentro de un sistema como el peruano, vive su propio proceso en el cual cohabitan individuos que responden a una serie de patrones socioculturales. El arraigo del contexto permite caracterizar el rol del docente cuando sobrepasa el límite de la autoridad y cae en el autoritarismo; sin embargo, se trata de comportamientos que justifican el rol del maestro. Dentro de esta perspectiva, se considera que el estudio es importante porque aporta, teóricamente, el deslinde entre las categorías del autoritarismo y la autoridad, relacionadas con el poder que tiene el docente; no obstante, se soslaya el poder del estudiante. Esto sirve para el análisis y el juzgamiento del desempeño docente dentro de la cultura que procura ser más democrática sin soslayar las propias expectativas.

En tal sentido, el estudio presentado por Huamán et. al, arroja los siguientes datos:

[d]el total de la población el $51 \%$ se encuentra en un nivel alto de autoritarismo, el $21 \%$ en un nivel moderadamente alto, el $15 \%$ en un nivel moderadamente bajo y el $13 \%$ en un nivel bajo de autoritarismo docente (2018, p. 69).

De esta manera, se evidencia que en el sistema educativo peruano existe un orden jerárquico establecido por el contexto social, donde el maestro somete al estudiante, determinando así el autoritarismo en su desempeño pedagógico. 
El presente estudio tiene como objetivo esclarecer la diferencia entre los conceptos de autoridad y autoritarismo dentro del salón de clases en el contexto peruano, haciendo uso de una encuesta elaborada en la plataforma de Google Forms, la cual fue respondida por docentes y estudiantes entre las edades de 15-60 años para ahondar en las perspectivas referente al tema de estudio. La elección de esta plataforma fue realizada debido al aislamiento social, producto de la emergencia sanitaria, COVID-19, ya que optando por la encuesta virtual se evitan aglomeraciones y posibles contagios.

Con la finalidad de contextualizar el estudio se observa que, a lo largo de las distintas épocas el uso y el abuso de la autoridad es aplicada para distintos fines: ya sea para guiar o controlar a las personas sin respetar sus ideales, afectando así el proceso de enseñanza aprendizaje (PEA). Esto se ha visto reflejado en la manera de educar buscando estudiantes sumisos, callados, dóciles que sean fáciles de manipular, pero las épocas van cambiando y la educación no es la excepción.

Históricamente hablando, aunque el ejercicio de poder tuvo orígenes muy remotos, cabe señalar que el estudio toma como referencia el periodo 1966 hasta 1979; la información se basa en el aporte de Muñasqui (2000), con la finalidad de focalizar la evolución del concepto de castigo en relación con el poder y por ende en la autoridad o autoritarismo.

En este recorrido, la Iglesia siempre consideró que los castigos corporales eran un método que conducía al estudiante a la salvación de su alma. La percepción de método infalible determinó, indirectamente, el poder de la autoridad de la Iglesia, así lo señaló Konstantinov (1966, citado por Muñasqui, 2000). Posteriormente, para Mayer (1969, citado por Muñasqui, 2000), la percepción del castigo se normalizó como un proceso natural y al mismo tiempo prevalecía la idea de Konstantinov al creer que es el único medio; no obstante, Merani (1969, citado por Muñasqui, 2000), aporta el cambio de percepción en el sentido de que la integridad de la persona se debe proteger a lo largo del proceso de formación académica, con ello se abandona el concepto de castigo como único medio. Cuatro años después, en 1973, el aporte de Ponce (citado por Muñasqui, 2000), el concepto dio un viraje que afirmaba que, en la educación primitiva, según la edad de piedra, se manifestaba una ausencia de coerción física o psicológica en los estudiantes. Con este aporte se cambió la perspectiva, con el fin de mirar la etapa inicial de Konstantinov. En cambio, para Foley (1979, citado por Muñasqui, 2000), el concepto se amplía en su percepción y surge el respeto hacia el estudiante, considerando que se trata de una ideología muy ligada al karma. El contexto señala las distintas percepciones acerca del castigo de un marco sociocultural; por ello, los siguientes cuestionamientos permiten situar la dicotomía entre el valor de la autoridad y el autoritarismo dentro de las prácticas educativas en el contexto de la escuela peruana, específicamente en la región de Lambayeque (Perú). Todo el proceso evolutivo del concepto y el apego a la construcción social en el que el escolar es protagonista del "método infalible" permite reafirmar el cuestionamiento, con el fin de situar la dicotomía como objeto de análisis; en tal sentido, se ratifica la siguiente interrogante: ¿Cuál es el constructo teórico que discrimina conceptualmente el autoritarismo y la autoridad en el contexto de una relación dicotómica?

La educación es uno de los procesos fundamentales para el desarrollo del ser humano; por lo tanto, todas las sociedades cuentan con un sistema educativo particular. En la educación peruana tanto el maestro como el estudiante se encuentran en el seno del proceso educativo, manteniendo una correlación docente-discente, la cual debe estar sujeta a un buen trato, respetando la autoridad del docente y la autonomía del alumno. 
El maestro debe ejercer su potestad como mecanismo necesario para guiar el proceso enseñanzaaprendizaje, pero sin caer en el autoritarismo, respetando la autonomía del estudiante. En este sentido, se busca analizar la relación dicotómica entre el adecuado ejercicio de autoridad y el autoritarismo en el salón de clases, como respuesta a la solución del caso presentado en el curso de Orientación y Bienestar del Educando del IV ciclo del programa de Lengua y Literatura, de la Facultad de Ciencias Histórico Sociales y Educación, de la Universidad Nacional Pedro Ruiz Gallo, Lambayeque (Perú).

El sentido crítico de las tres preguntas ha permitido comprender, dentro de las perspectivas, que ser autoritario puede ser plausible en tanto el contexto lo permite; sin embargo, el autoritarismo puede sobrepasar el límite que contempla el proceso formativo del individuo dentro del sistema educativo. De este modo, en el estudio se define que el sentido dicotómico es una oportunidad para el docente, con la finalidad de mejorar los procesos que conducen el desarrollo humano mediante el pensamiento crítico sin soslayar la inteligencia emocional. Finalmente, y sin darle el espacio de privilegio al concepto de autoridad, tampoco a la idea de autoritarismo, el estudio tiene como objetivo: analizar la relación dicotómica entre el adecuado ejercicio de la autoridad y el autoritarismo en el salón de clases.

\section{METODOLOGÍA}

El proceso metodológico del estudio aborda diferentes perspectivas ahondando en experiencias individuales con respecto a la autoridad y autoritarismo (expresado como una breve anécdota planteada por un alumno), que a su vez presenta dos elementos cualitativos que analizar, como es el caso del maestro y el estudiante. Durante el desarrollo del curso de Orientación y Bienestar del Educando, correspondiente al V ciclo del Programa de Lengua y Literatura, de la Escuela Profesional de Ciencias de la Educación de la Universidad Nacional Pedro Ruiz Gallo.

La Chakana pregunta es la estrategia didáctica que se basa en el planteamiento de nueve preguntas, con la finalidad de redactar textos académicos. Las nueve preguntas se ubican en la Chakana, la misma que representa la cosmovisión andina. El planteamiento de las nueve preguntas desarrolla el proceso evolutivo de la cognición hasta llegar al pensamiento crítico. Una vez logradas las preguntas, se desarrollan seis niveles de análisis: horizontal, vertical, diagonal, convergente, divergente y el circular.

El análisis categorial y las implicancias de la dicotomía se discuten mediante tres cuestionamientos, como producto del análisis del caso. Estas tres interrogantes se ubican en la parte central de la Chakana pregunta (figura n. ${ }^{\circ}$ 1), con la finalidad de darle sentido al objetivo planteado. Las tres interrogantes no buscan respuesta; por el contrario, se trata del análisis del cuestionamiento y las implicancias cuando el proceso de aprendizaje se desprende de un docente autoritario que presume autoridad, por ello las preguntas: “¿Qué significa ser un maestro autoritario?”, “¿Cuán efectiva es la enseñanza de un maestro autoritario?” y “¿Cómo ejercer la autoridad sin transgredir la libertad del alumno?”. Las tres interrogaciones se presentan como eje del contenido analítico del estudio. Se trata de tres ejes para el análisis que lo único que busca es el equilibrio emocional del docente para saber en qué momento debe demostrar sabiduría para ello. Las tres preguntas (análisis vertical-central) han servido para el estudio de las interrogantes sin pretender respuestas. Este ejercicio permite determinar la pertinencia de la Chakana pregunta como estrategia para 
producir textos, el análisis vertical no condiciona a los autores, sino que permite ejercer la libertad y autonomía del equipo para tomar decisiones.

Técnicas de recogida de datos

Después del análisis del caso antes mencionado se disponen nueve preguntas según el prototipo andino; sin embargo, para el estudio, se ha utilizado tres preguntas que reflejan pensamiento crítico (análisis vertical-central). Se seleccionó el análisis vertical, para así explicar los tres cuestionamientos, sin pretender respuestas que permitan la reflexión y análisis del caso. Para su desarrollo y la obtención de resultados, se ha empleado una encuesta mediante Google Forms (cuestionario), la muestra es de 105 personas entre las edades de 15-64 años aproximadamente. el $64.76 \%$ responden al género masculino, el $34.28 \%$, del género femenino, el $0.95 \%$ de la muestra prefirió no indicar su género. La búsqueda de información sirvió para situar, en contexto, las diversas perspectivas en torno a la relación dicotómica entre autoridad y autoritarismo, razón por la cual la discusión empieza por la pregunta fundamental (PF). Al ser un trabajo colectivo, se recogieron las opiniones de los investigadores - referencias autobiográficas - porque se trata de un caso real, con la finalidad de desarrollar el pensamiento crítico en torno a la relación dicotómica entre autoritarismo y autoridad.

La forma de abordar el problema a partir del caso planteado tiene dos perspectivas: la discusión de la dicotomía autoridad-autoritarismo-autoridad, pero al mismo tiempo la vivencia de la Chakana pregunta como metodología para producir textos académicos, toda vez que el desarrollo del sistema de capacidades para redactar es muy incipiente durante el proceso formativo para ser docente. Por lo tanto, la metodología empleada se basa en el enfoque cualitativo porque recoge las perspectivas en cuanto a la solución del caso, pero al mismo tiempo la vivencia de la redacción académica utilizando la Chakana pregunta.

El estudio tiene énfasis en la caracterización de la Chakana pregunta como estrategia didáctica que desarrolla el pensamiento crítico utilizando solo tres preguntas de nueve como consecuencia de la valoración del caso planteado.

La metodología empleada contribuye a la investigación porque sigue un propósito teórico en cuanto al análisis dicotómico entre autoridad-autoritarismo del docente en el caso establecido, puesto que explica la relación causa-efecto. Asimismo, la Chakana pregunta ha servido como base dialéctica que ha permitido analizar el caso mediante la elaboración de nueve preguntas y la selección del análisis vertical, el mismo que se define como "el recorrido cognitivo y analítico que sucede desde la pregunta complementaria [PC] hasta la pregunta fundamental [PF] para vincularse a otra [pregunta] complementaria [PC]. Este recorrido puede desarrollarse en sentido inverso durante todo el proceso analítico" (Bocanegra, 2020, p. 244). Entonces, se registran dos eventos principales: contribuir a la solución del caso mediante el planteamiento de nueve preguntas y la discriminación de tres de ellas para redactar el presente texto académico en el que se analiza la relación dicotómica: autoritarismo-autoridad.

El empleo de la metodología se justifica porque genera el análisis de un caso real dentro del proceso formativo profesión y utilizar una estrategia didáctica como la Chakana pregunta. Constituye un gran aporte al desarrollo de capacidades y se justifica porque se logra evidenciar habilidades como observar, analizar, interpretar, inferir, observar, exponer y regular ideas, cuestionamientos, 
posturas morales y racionales; todo ello llevado a la práctica de la elaboración de nueve preguntas $y$, al mismo tiempo, asumir el reto de seleccionar solo tres con la finalidad de redactar el texto académico (Figura 1).

Figura 1. El análisis vertical central en la Chakana pregunta

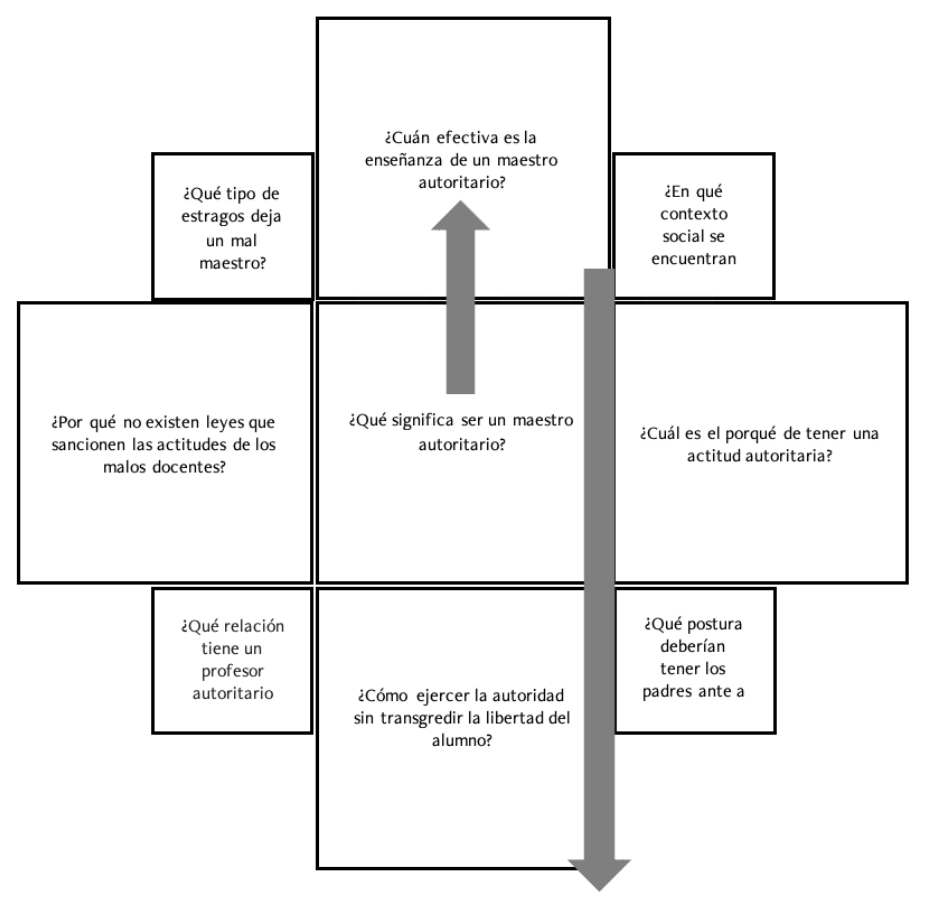

Fuente: Elaboración propia con base en la estrategia didáctica de la Chakana pregunta.

Se evidencia el análisis vertical central, el mismo que se inicia en la pregunta fundamental (PF), se traslada a la pregunta principal (PP) y concluye en a pregunta principal (PP) inferior.

\section{DESARROLLO}

El maestro es quien origina un buen ambiente escolar y debe saber cómo emplear su autoridad dependiendo de las diversas situaciones que se suscitan dentro del salón de clases. Tal como expresa Pierella, “[s]e ha señalado el carácter necesario de la autoridad en las relaciones pedagógicas y la necesidad de volver a pensarla, cuestionando los modos tradicionales de ejercerla” (2014, p. 895). Es decir, que las reglas son necesarias para el entorno escolar, pero estas no deben coaccionar la libertad de los estudiantes, dejando en claro que su propósito es establecer un espacio ordenado en donde enseñar y aprender sea más factible.

En el entorno escolar, la figura en la que recae la autoridad es el maestro y este se encarga de direccionar a los estudiantes. Por lo tanto, se puede decir que el docente es quien brinda la instrucción necesaria a cada estudiante para descubrir sus distintas habilidades y formas de aprender. Esto se logra gracias al buen uso de la autoridad del maestro, ya que este vislumbra el camino para desarrollar mejor su proceso de aprendizaje de forma personalizada. Según Bourdieu \& Passeron (1996, citado por Meza et ál., 2017), “[l]a autoridad pedagógica se entiende como la relación asimétrica que establece el profesor con los alumnos que le permite influir sobre ellos y conseguir que acepten sus demandas y orientaciones en el ámbito de la enseñanza y aprendizaje" (p. 222). 
En un primer momento, el hombre primitivo consideraba su entorno como fuente principal de aprendizaje (mimética), ya que todo era nuevo para él, por este motivo la educación aún no era escolarizada, dando lugar a que los líderes de las tribus se encargaran de difundir los conocimientos empíricos. De acuerdo con Ponce (1973), “[...] durante el aprendizaje los niños no eran nunca castigados” (p. 9). Esto nos da a entender que las personas encargadas de direccionar la educación consideraban importante aprender a partir del ensayo y error, evitando así el castigo corporal.

Siglos después, en el Medio Oriente, específicamente en Egipto, la educación tiene variaciones, porque alcanza un nivel escolarizado, dando lugar a los primeros maestros, denominados escribas, los cuales aplicaban supremacía sobre sus estudiantes de manera física, psicológica e intelectual. En este sentido, Mayer (1967, citado por Muñasqui, 2000) manifiesta: “[t]ú me golpeaste y el saber se penetró en mi cabeza. El maestro, por su parte, decía: tú eras para mí un asno; yo haré de ti un hombre" (p. 89). Por otro lado, en la cultura hindú se puede observar una diferencia relevante entre esta civilización y la ciudad de los faraones, puesto que los alumnos no estaban sometidos a constantes maltratos, por estar respaldados por el Código de Manú, el cual destaca la educación sin castigos, pero los maestros hacían prevalecer su autoridad por medio del miedo. En tal caso, Foley (1979, citado por Muñasqui, 2000) resume: “[e]l que ofende a un maestro pasará después de muerto al cuerpo de un asno” (p. 65). En la Edad Media, la sociedad estaba sometida por la Iglesia la cual se encargaba de educar a las personas, pero son los niños los más influencia dos por dicha educación canónica.

Konstantinov (1966, citado por Muñasqui, 2000) dice:

El maestro no perdona los errores de sus alumnos; la aplicación de castigos crueles estaba muy extendida. Esto era aprobado por la Iglesia que sostenía que la naturaleza del hombre es pecaminosa y que los castigos corporales contribuyen a la salvación del alma, pues expulsan de ella el principio diabólico (p. 30).

Dentro del panorama eclesiástico, se puede notar el abuso de autoridad para lograr sumisión en los alumnos, todo esto difundido por el miedo, teniendo como objetivo suprimir el pensamiento crítico y manipulando el conocimiento.

En la Edad Moderna, la educación era en su totalidad escolarizada destacando las escuelas públicas y privadas, siendo las primeras financiadas por el Estado. Dentro de las escuelas, los maestros ya tenían plena autonomía para direccionar el aprendizaje de sus estudiantes, lo que permitió la creación de una figura de autoridad dentro de las aulas. En ese sentido, Merani (1969, citado por Muñasqui, 2000) expresa:

El maestro es guía, sostén, consejero, compañero, hermano y padre que respeta el carácter y ayuda a formar la personalidad de aquellos que le fueron confiados. La enseñanza, la disciplina, la educación, deben convertirse en un auto aprendizaje, autogobierno, autoeducación (pp. 41-42).

En el contexto actual, el proceso educativo nos presenta al estudiante y al profesor en un mismo orden jerárquico, cambiando así la perspectiva común de mantener un margen diferenciador entre 
estos dos, llegando a destacar ambas partes como elementos principales del proceso formativo de enseñanza-aprendizaje (PEA). Tal como se observa en épocas anteriores el profesor recurre al castigo físico e incluso psicológico; pero hoy en día cualquier tipo de abuso a la integridad del estudiante es penado, en el caso peruano el alumno cuenta con el respaldo de normas legales para protegerlo en caso de ir en contra de sus derechos. En tal sentido, el Decreto Supremo n. ${ }^{\circ}$ o03-2018-MIMP (2018), dice lo siguiente:

El castigo físico y humillante es incompatible con la educación, el respeto a la dignidad humana de la niña, niño y adolescente y los límites estrictos de la disciplina escolar. El/ La docente, ante un mal comportamiento, debe emplear la comunicación con las niñas, niños y adolescentes de acuerdo a sus características, ayudándoles a desarrollar la responsabilidad de sus actos, apoyándose en la evidencia de las razones, y la disciplina escolar (p. 31)

\section{1 ¿Qué significa ser un maestro autoritario?}

En la encuesta elaborada por Lizeth Caramantín, Josué Mejía y Guadalupe Zuñe en la plataforma de Google Forms, titulada "Autoridad vs. Autoritarismo", con una muestra de 105 personas que dieron respuesta a la primera interrogante "¿Conoce usted la diferencia entre ejercer autoridad y autoritarismo?”. En consecuencia, de sus respuestas de afirmación o negación a la cuestión planteada, el 86.7 \% respondió que sí conoce dicha diferencia y el otro 13.3 \% la desconoce; entonces, se infiere que la gran mayoría de los participantes expresan un conocimiento superficial entre los conceptos de autoridad y autoritarismo.

Así mismo, el estudio que realiza Miranda nos demuestra lo siguiente:

Al hacer el análisis por país encontraron los mismos resultados para Perú. Bardales y Huallpa (2005) llevaron a cabo una investigación en tres departamentos del Perú y hallaron que el $13 \%$ de niñas y niños había sufrido de maltrato físico en la escuela, y que el $98 \%$ de ellos señaló que era el profesor quien ejercía este maltrato (2016, p. 4).

A la misma muestra de personas se les planteó la siguiente pregunta: “¿Ha tenido usted algún maestro/a autoritario/a?", teniendo como respuesta que un abrumador $73.3 \%$ afirma haber presenciado las actitudes de un maestro autoritario, pero un $26.7 \%$ alegan no haber tenido contacto con este tipo de maestros. Dejando en claro la cuestión “¿Qué definición tienen las personas encuestadas con respecto al concepto del maestro autoritario?”, muchas veces, no se logra distinguir claramente entre un maestro que ejerce su autoridad y otro que abusa de esta. Al respecto, Peiró-i et ál. consideran que “[c] onviene distinguir que el autoritarismo se manifiesta como defecto en el ejercicio del poder, pues se basa en un poder arbitrario donde alguien se erige en el lugar de la Ley" (2019, p. 267). Es decir, el docente no permite contradicciones ni acepta aportes, ya que se considera el único con derecho a transmitir conocimientos verídicos, tomando cualquier tipo de cuestionamiento o participación como falta de respeto a su capacidad e intelecto.

Según Muñoz (2012), “autoridad no es equivalente ni a control ni a obediencia, esta tiene un sentido de reconocimiento y legitimidad que puede generar el control o la obediencia, pero estos 
no siempre son consecuencia de la autoridad" (citado por Gallego et al., 2016, p. 121). En este sentido, se cuestiona el derecho a ejercer autoridad en los centros educativos, enfatizando que es un atributo el cual se debe ganar y no imponer. Por lo tanto, el profesor debe encontrar la manera más adecuada de relacionarse con sus alumnos, tratando de establecer un buen clima institucional y sosteniéndose en una comunicación recíproca, clara y directa; de esta manera, se pueden solucionar los conflictos que se presenten, para incentivar un adecuado desarrollo del proceso educativo.

Se cuestiona la dicotomía propugnada por Muñoz, porque el debate del concepto define el rol del maestro, el sentido ecléctico no ayuda a comprender y valorar el rol del docente porque no se establece el límite tanto de alumno como del educador. La disputa del poder depende mucho del sentido de autoridad, dentro del espectro de la escuela rural es posible que un escolar tenga más autoridad que el docente; por lo tanto, la autoridad equivale al poder que ejerce quien lo domina, pero al mismo tiempo engendra obediencia.

\section{2 ¿Cuán efectiva es la enseñanza de un maestro autoritario?}

En la encuesta elaborada por Lizeth Caramantín, Josué Mejía y Guadalupe Zuñe en la plataforma de Google Forms titulada: “Autoridad vs. autoritarismo”, también se planteó la siguiente pregunta: “¿Prefiere usted un maestro/a sin autoridad que enseñe mal o un maestro/a autoritario/a que enseñe bien?". Las respuestas de las 100 encuestados revelaron un dato importancia que gira en torno a los estudiantes, el $94 \%$ prefiere que se les enseñe bien, aunque sea autoritario; mientras que el $6 \%$ prefiere un docente sin autoridad, aunque enseñe mal. Existe un tipo de maestro que se muestra muy ameno con sus educandos, optando por no desarrollar una buena sesión de aprendizaje y solo se dedica a perder el tiempo, ya sea leyendo un periódico, revisar su teléfono celular o contando anécdotas que no aportan al aprendizaje del alumno. Esta actitud genera en el escolar un concepto sobre el educador, tomándolo como referencia para optar por una actitud despreocupada frente a la escuela; ante a esto, la actitud de los estudiantes difiere entre la adaptación al bajo nivel recibido por parte del maestro o la preocupación por obtener un aprendizaje significativo, siendo este último el tipo de estudiante que más prima con un $94 \%$ de aceptación a tener un maestro autoritario que haga prevalecer una enseñanza sustancial, ante un $6 \%$ que prefiere a un maestro sin autoridad con un mal modelo de enseñanza.

Al respecto, Hidalgo (2020) opina que "[h]acer autoridad es la autoridad de la capacidad, es decir, la capacidad del autor de permitir, aumentar, enriquecer, desarrollar (augere) la autonomía en la otra persona" (p. 260). De tal manera que toda habilidad social se desarrolla de manera progresiva y el adecuado ejercicio de la autoridad no difiere de este enfoque. Asimismo, en la encuesta realizada mediante Google Forms, marzo de 2021, una de las experiencias menciona: "Una profesora en clase trató de imponernos sus ideas sobre un partido político, mencionaba que era la mejor y única opción. Yo intervine diciendo que cada uno es libre de investigar y de pensar bien sobre a quién apoyar". El caso demuestra que muchos docentes imponen de manera total o parcial sus ideas, basándose en la autoridad que posee y haciendo mal uso de esta, una manera correcta de actuar de la docente debe tener en cuenta la libertad de expresión de su alumno. No obstante, las perspectivas sobre el mismo constructo son variadas, nótese en el siguiente testimonio: 
No tiene nada de malo tener autoridad, lo que sí está mal es abusar de esta, no tuve problemas en el colegio, pero sí en la universidad, en una ocasión tuvimos una situación en la que el profesor hizo mal dos preguntas del examen, fue su error, después, en la siguiente clase, mis compañeros reclamaron, le pidieron que tenga en consideración esas dos preguntas, pero el profesor no quiso hacerlo y dio a entender que no tendría en consideración esas preguntas "porque él lo decía”. (E. Céspedes, comunicación personal, 27 de marzo de 2021)

Entonces, se observa cómo la educación basada en el abuso de autoridad restringe la libertad del alumno; en tal caso, si el docente realizara un buen ejercicio de su autoridad, tendría la posibilidad para asumir sus errores y admitir que los estudiantes tenían razón al exigir la corrección del examen, de ser así, el resultado sería distinto. Al respecto, Duarte y Abreu (2014) sostienen que “[c]uando la autoridad promueve la libertad, se da dentro de las relaciones personales, se realizan actividades como el cuestionamiento, el entendimiento, la comprensión; el aprendizaje se vuelve significativo" (p. 99). La autonomía que poseen los estudiantes para cuestionar ciertas actitudes de sus maestros no debe reprimirse, porque muchas veces las decisiones asumidas por los docentes perjudican no solo el aprendizaje, sino también el entusiasmo y la imaginación de los alumnos, privándoles su derecho a expresar sus ideas sin temor a ser juzgados ni considerados como mal educados.

\section{3 ¿Cómo ejercer la autoridad sin transgredir la libertad del alumno?}

De los encuestados por Lizeth Caramantín, Josué Mejía y Guadalupe Zuñe, 96 personas dieron respuesta a la siguiente pregunta: “¿Cuál de las siguientes características cree usted que posee un maestro/a autoritario/a?". Como resultado, el $69.8 \%$ considera que un maestro autoritario es aquel que tiene el tono de voz alto, mantiene el orden del salón y es estricto en la presentación de trabajos; por otro lado, un $30.2 \%$ manifiesta que un maestro que ejerce autoritarismo debe tener estas actitudes: impone sus ideas, usa el castigo e imposibilita la elección; en consecuencia, se puede observar la gran confusión que existe al identificar una actitud autocrática del docente, siendo un error común tanto en estudiantes como en profesores que no distinguen las características que forman parte de un abuso de autoridad. Para tener un concepto más claro acerca del tema, García y Mendoza (2009) expresan que "[e]l autoritarismo docente nos ha dicho que el docente enseña y el alumno acata; que uno lo sabe todo y el otro nada; que el docente decide y opera y el alumno se silencia y obedece" (p. 72). La educación clásica manifiesta que un maestro serio, impositivo y castigador presenta características relacionadas con la buena educación, lo cual es erróneo, pues esto no permite el interés genuino del estudiante por aprender, sino que, al contrario, estas características limitan al educando y generan una antipatía hacia el aprendizaje.

Asimismo, existen estudios de casos que demuestran ciertos comportamientos. Por ejemplo, el $69.8 \%$ considera que un docente debe tener un tono de voz alto, mantener el orden del salón y ser estricto en la presentación de trabajos; mientras que el 30.2 \% considera que el docente debe imponerse con sus ideas, emplear el castigo y no dar posibilidad de elección. Los hallazgos solo representan una diversidad de conceptos que permite favorecer el comportamiento etológico del docente o declararse antípoda a la idea; por ello, la discusión dicotómica es contundente: “puesto que el conocimiento antecede y precede al concepto" (Bocanegra, 2021, p. 263). Por lo tanto, el autoritarismo solo puede pervivir donde es permisible, la autoridad debe servir para 
comprender la naturaleza humana desde una escuela con autoridad. En esta misma tendencia, de los 105 encuestados se registraron 86; es decir, el 81.9 \% planteó situaciones anecdóticas que reflejan distintas percepciones acerca de la dicotomía autoritarismo-autoridad. Véase el siguiente registro:

Tuve una maestra en secundaria que imponía su opinión en los temas con respecto a su curso y si alguien estaba en desacuerdo con su opinión no la respetaba y hacía abuso de poder pues tenía varios cargos en el colegio y a las estudiantes que no compartían su opinión luego les bajaba la nota sin razón alguna o afectaba la nota de conducta de ellas. (A. Vásquez, comunicación personal, 26 de marzo de 2021)

Sin embargo, otro de los encuestados, expresa su opinión al respecto señalando:

Un docente de la universidad que me enseñaba el curso de Economía de la Educación era muy estricto, serio, pero no nos imponía sus ideas. Solo quería que los estudiantes estén atentos a clase (C. Bautista, comunicación personal, 26 de marzo de 2021).

Para esclarecer la dicotomía que existe acerca del tema, se define que "la autoridad se aleja del autoritarismo en la medida en que es reconocida y aceptada voluntariamente" (García, citado por Plaza, 2018, p. 270). En tal caso, es primordial que los estudiantes reconozcan una figura de autoridad, tomando en cuenta que ellos también son responsables de exigir cómo se debe orientar el aprendizaje significativo; por lo tanto, se necesita respetar los derechos tanto del alumno como del profesor y tener en claro sus obligaciones de acuerdo con el rol que cumplen. En consecuencia, aceptar la autoridad no es someterse ante una representación de poder, sino más bien reconocerla, de lo contrario, estaríamos hablando de autoritarismo, el mismo que determina una serie de actitudes y comportamientos negativos del docente, sin considerar la aprobación o aceptación del alumno, buscando someter el pensamiento, el conocimiento y los principios de los estudiantes.

\section{CONCLUSIONES}

En el análisis reflexivo de la información, existe una delgada línea entre ejercer autoridad y abusar de la misma. Es necesario que la autoridad establezca orden, con el fin de estimular y guiar a los estudiantes a mejorar el proceso enseñanza-aprendizaje. El análisis de la dicotomía tiene algunas limitaciones porque no se reconoce el papel principal del docente cuando debe someter al estudiante a un papel pasivo o al cultivo de la obediencia para mejorar la convivencia escolar enfocada en las necesidades educativas y significativas del estudiante.

En el acto educativo, los docentes deben sustentar su actuación en un concepto absoluto de autoridad para ejercerla de forma adecuada en el salón de clases, tomando en cuenta la libre expresión, la autonomía y su participación en el aprendizaje. Por lo tanto, el estudio abre otras líneas de investigación orientadas a mejorar la autoridad del docente sin llegar al autoritarismo porque el rol es una manifestación etológica frente al poder de la autoridad, muchas veces sin tenerla. 
La relación dicotómica establecida entre la autoridad y el autoritarismo en la práctica educativa ha permitido determinar que el desempeño docente debe ser la demostración de muchas competencias como respuesta a situaciones inesperadas, lo que permite suponer que la inteligencia emocional es una característica ontológica del hombre, sobre todo si se trata de la formación humana.

Las limitaciones existentes para la elaboración del trabajo fue la falta de información sobre el tema de autoridad y autoritarismo, en específico, los datos estadísticos que evidencian la problemática del tema de estudio.

\section{REFERENCIAS}

Bocanegra, B. (2021). El exante y expost del pensamiento crítico de la Chakana Pregunta. Revista Redipe, 10(1), 259-71. https://doi.org/10.3626o/rbr.v10i1.1178

Duarte, A., \& Abreu, J. (2014). La Autoridad, Dentro del Aula; Ausente en el Proceso de Enseñanza Aprendizaje. Daena, International Journal of Good Conscience, (2), 90-121. http://www.spentamexico.org/ v9-n2/A9.9(2)90-121.pdf

Decreto Supremo 003-2018-MIMP, Ley que prohíbe el uso del castigo físico y humillante contra los niños, niñas y adolescentes. 7 de junio de 2018. https://cutt.ly/2WxXc9O

Gallego, L., Acosta, J., Villalobos, Y., Lopez, A., \& Giraldo, A. (2016). La violencia docente en el aula de clases. Revista de Investigaciones UCM, (28), 116-125. http://dx.doi.org/10.22383/ri.v16i2.81

García, Y. \& Mendoza, B. (2009). El autoritarismo y su expresión en la formación docente. Laurus, (15), 71-93. https://cutt.ly/BWxXECP

Hidalgo, K. (2020). Incomprensión o legitimación del concepto de Autoridad. ¿Qué se busca en Ecuador? INNOVA Research Journal, 1(6), 257-272. https://doi.org/10.33890/innova.v6.n1.2021.1487

Meza, M; Zamora, G., \& Cox, P. (2017) Ejercicio de la autoridad en profesores de educación secundaria de Chile. Estudios Pedagógicos, XLIII(2), 221-235. https://scielo.conicyt.cl/pdf/estped/v43n2/art12.pdf

Miranda, A. (2016). El uso del castigo físico por parte del docente, y el rendimiento de los estudiantes en la sierra peruana. Grupo de Análisis para el Desarrollo. http://www.grade.org.pe/wp-content/uploads/ai21.pdf

Muñasqui, T. (2000), Génesis y Desarrollo del Paradigma Educativo Enfoque dialéctico. Editorial San Marcos.

Peiró-i, S., Beresaluce, R., \& García, M. (2019) Autoridad, liderazgo y convivencia en el aula. Revista Observatório, (5), 256-291. http://dx.doi.org/10.20873/uft.2447-4266.2019v5n2p256

Pierella, M. (2014). La autoridad de los profesores desde la perspectiva estudiantil. Revista Brasileira de Educação, 57(19), 893-912. https://www.redalyc.org/pdf/275/27532619005.pdf

Plaza, J. (2018). Autoridad docente y Nuevas Tecnologías: cambios, retos y oportunidades. Revista complutense de Educación, 29(1), 269-286. http://dx.doi.org/10.5209/RCED.52281

Huamán, J., \& Zambrano, E. (2018). El docente autoritario y su relación con el rendimiento escolar del área de historia, geografía y economía de los estudiantes del tercer grado de secundaria de la I. E. Juan Domingo Zamácola y Jáuregui del distrito de Cerro Colorado, Arequipa. (Tesis de grado, Universidad Nacional de San Agustín de Arequipa). Repositorio Institucional. http://repositorio.unsa. edu.pe/handle/UNSA/6038 


\section{AUTORES}

Guadalupe del Pilar Zuñe Flores. Estudiante de pregrado de la Universidad Nacional Pedro Ruiz Gallo de la facultad de Educación de la especialidad de Lengua y Literatura

Josué Joel Mejía Guevara. Estudiante de pregrado de la Universidad Nacional Pedro Ruiz Gallo de la facultad de Educación de la especialidad de Lengua y Literatura.

Lizeth del Milagro Caramantin Castillo. Estudiante de pregrado de la Universidad Nacional Pedro Ruiz Gallo de la facultad de Educación de la especialidad de Lengua y Literatura.

Beder Bocanegra Vilcamango. Magister en Gerencia Social por la Universidad Nacional Pedro Ruiz Gallo, Lambayeque-Perú. Docente de la Escuela Profesional de Ciencias de la Educación, de la Universidad Nacional Pedro Ruiz, Lambayeque-Perú.

\section{Conflicto de intereses}

Los autores informan que no existe conflicto de interés posible.

\section{Financiamiento}

No hubo asistencia financiera de partes externas al presente artículo.

Agradecimientos

Expresamos nuestro agradecimiento a: Alexander Mejía, América Guevara, Camila Caramantin, Carlos Caramantin, Carlos Zuñe, Dany Zuñe, David Zuñe, Elmer Mejía, José Gerson Alama Nunura, Lourdes del Milagro Zuñe Flores, Marianela Flores Chanamé, Rosa Matilde Castillo Amaya. 